\title{
Multifractal Scaling Law Versus Gradient Elasticity in the Evaluation of Disordered Materials Compressive Strength
}

\author{
G. Efremidis ${ }^{1}$, A. Carpinteri ${ }^{2}$ and E. C. Aifantis ${ }^{1,3}$ \\ 'Laboratory of Mechanics and Materials, Polytechnic School, Aristotle University of Thessaloniki, \\ GR-54006 Thessaloniki, Hellas \\ ${ }^{2}$ Politecnico di Torino, Department of Structural Engineering, Corso Duca degli Abruzzi 24, \\ 1-10129 Torino, Italy \\ ${ }^{3}$ Center for Mechanics of Materials, Michigan Technological University, \\ Houghton MI 49931, USA
}

\begin{abstract}
It is shown that the phenomenon of strength dependence on size, for otherwise geometrically similar specimens, may be interpreted on the basis of gradient elasticity or multifractal scaling law arguments. This paper compares the predictions of these two different approaches to the scaling properties of the compressive strength in disordered materials. A detailed study for the stress concentration factor and the normalized failure stress of the borehole axisymmetric plane strain problem is presented. This problem has been already solved analytically by using a simple form of gradient elasticity theory. However the solution was not explored sufficiently to illustrate the size effect in a bilogarithmic diagram. By doing this the predictions of gradient elasticity can be related to the predictions of the multifractal scaling law. A comparison between the two mentioned approaches is presented and a common denominator is found.
\end{abstract}

\section{INTRODUCTION}

Many physical phenomena can be analyzed through classical continuum mechanics, whenever one length or energy scale (i.e. macroscopic scale) is relevant. When a critical phenomenon is imminent, other scales of: observation become also relevant, for instance in material failure. These are co-operative and multiscale phenomena; then, the global behavior cannot be predicted simply by applying a local law. Usually, a rather rough distinction between nano-, micro-, meso-, and macroscopic scales is made, although the scaling ranges are in actual fact infinite and not discrete. From a practical point of view, we can pass from one observation scale to another without accounting explicitly for any discontinuity. Classical continuum mechanics cannot always interpret phenomena like material damage, strain localization and fracture. It is also impossible to 
explain the size effects on tensile and compressive strength, when uniaxial tests (tensile or compressive) on initially uncracked specimens are considered. The novel concepts of disorder, fractality and renormalization group theory have been considered in order to describe the above phenomena in a unified manner $/ 1$.

The dominant mechanism of brittle fracture in compression is basically Mode I cracking as in tension. Even under all-around compressive loads, brittle materials tend to fail by the formation of tensile cracks at microdefects such as flaws, cavities, holes, inclusions and grain interfaces $/ 3 /$. Tensile strains are parallel to the applied load direction with tensile loads, or perpendicular to the applied load direction with compressive loads. In uniaxial compression, macroscopic cracks start parallel to the direction of axial loading. The only realistic explanation for this behavior is provided by the intrinsic heterogeneity of the material, and especially by the presence of cracks, pores and voids even prior to the application of the load. The pre-existing defects act as stress-raisers and they strongly reduce the strength of the material $/ 3 /$.

The two potential sources of disordered damage patterns are the intrinsic instability of the cracking process and the effect of the random pre-existing material inhomogeneities. It can be noted that the main source of randomness and chaotic behavior is the inherent disorder of the material microstructure. Whenever entropy wins over energy, the resulting structure will be dominated more by randomness than by strict Euclidean order and we might expect to find fractal patterns with self-similar scaling symmetry (Mandelbrot, 1982), 14/. The application of fractal geometry to concrete-like materials has proven to be very appropriate, due to the multi-scale heterogeneity of these materials $/ 5 /$. This is reflected into the hierarchical process of failure. The interfacial zone between cement paste and aggregates is one of the weakest in the concrete microstructure. Cracks usually originate from pre-existing flaws (pores) in the matrix or at interfaces (microlevel). Afterwards, they develop at the scale of the matrix (meso-level), and finally extend to the structural size scale (macro-level), resulting into a typical disordered pattern with multiple cracking randomly diffused at the interfaces or through the matrix and the aggregates. This propagation process often leads to wellorganized patterns at larger scales.

The presence of an upper and a lower bound in the scaling range produces an inevitable transition from the fractal (disordered) regime at the microscopic scale to the Euclidean (homogeneous) regime at the largest scales. The upper bound is represented by the macroscopic size of the specimen, whilst the lower one is related to the size of the smallest particles (grains for metals, crystals for rocks, aggregates for concrete). The presence of this internal length, typical of any microstructure, inhibits the development of a perfect selfsimilar scaling through the whole scale range $/ 5 /$. Mathematical fractals, lacking any characteristic length, exhibit uniform (monofractal) scaling without any bound and can be characterized by a unique value of the fractal dimension.

\section{GENERAL ASPECTS OF GRADIENT THEORY AND MULTIFRACTAL SCALING LAW}

\subsection{Gradient approach}

Gradient theory has been introduced by Aifantis and his co-workers in the early 1980's in order to address the problem of dislocation patterning, to provide estimates of the shear band thickness, and settle the issue of 
mesh-size dependence of finite element calculations in the strain softening regime. An additional problem that was possible to address within the setting of gradient theory is concerned with the bridging of length scales. This approach captures the occurrence of material instabilities at the microscale and predicts their effect on the macroscopic response. Various applications and example problems have been worked out for dislocation patterning at the mesoscale, localization of deformation at the macroscale, wave propagation, surface instabilities, size effects, damage and fracture. The aforementioned research activity on gradient theory has produced results related to unresolved problems in modern technology, including the material/manufacturing and the concrete/construction industries. Such results are expected to be useful in other industrial and natural processes, including microelectronics/thin film technology, drilling/penetration, and hazard/earthquake mitigation, where the interaction of length scales are critical factors in the understanding and controlling of the evolution of the process.

The gradient approach enriches the standard equations of solid mechanics with an internal length scale, thus making them amenable to nonlinear stability analysis in the strain softening regime. In fact, the only material length implicitly involved in the governing equations of classical continuum mechanics is the interatomic distance which is not possible to utilize for explaining the overabundance of deformation patterns observed at various scales, ranging from nanometers (dislocations) and micrometers (slip bands), to millimeters (shear bands) and kilometers (faults, avalanches).

The need for higher order gradients in the theory of deformation for softening solids and heterogeneous microstructures was first pointed out by Aifantis (1983) $/ 6-8 /$, in relation to the problems of shear band thickness and persistent slip band spacing. Higher order theories in elasticity have been proposed since the beginning of the century in order to account for the effect of microstructure. However, their implications to deformation patterning and size effects have not been explored. It also emphasized that classical continuum mechanics theories cannot describe situations dominated by microstructural effects, e.g. load or geometrically induced stress singularities. Higher order theories play an important role in eliminating such singularities and interpreting various deformation and crack patterns associated with failure.

In particular, gradient elasticity was used to eliminate singularities or discontinuities of classical elasticity such as those occurring at crack tips and dislocation cores. Altan and Aifantis $/ 9 /$, for example, examined the structure of the crack tip for Mode III loading conditions and illustrated that the strain is finite at the crack tip with the only non-vanishing displacement component satisfying Barenblatt's crack closure condition. By considering that the crack tip is unclamped and using the Fourier transform technique, they found that, in contrast to the prediction of the classical theory, the crack lips close sharply giving a cusp-like crack profile. This property of the solution indicates that gradient elasticity is consistent with Barenblatt's cohesive zone theory, without requiring the extraneous assumption on the existence of interatomic cohesive forces, whose effect is replaced accounted for here in the constitutive equation through by the gradient term. It was also shown that, as the gradient coefficient $c$ tends to zero, the solution approaches the classical one. In contrast, the stresses remain the same as in the classical theory for traction boundary-value problem. In a follow-up paper Ru and Aifantis /10/ outlined a procedure for obtaining solutions of certain boundary value problems in gradient elasticity, in terms of corresponding solutions of classical elasticity. The method was applied to 
illustrate how the gradient theory can remove the strain singularity associated with some typical examples of the classical theory (Flamant problem), as well as to handle interface problems and, in particular, to remove the "overlapping condition" appearing in the solution of interface cracks. Different solutions for Mode III were obtained by Unger and Aifantis /11/ by using different boundary conditions and different signs for the gradient coefficient $c$. Among the results obtained in $/ 10,11 /$ are also those concerned with the derivation of different types of crack profiles including oscillatory ones. Exadaktylos and Aifantis $/ 12 /$ have also used Gradient Elasticity to obtain analytical asymptotic solutions of mixed boundary value problems for Mode I,II,III configurations, as well as for penny-shaped clamped-tip cracks, by applying special integral transform techniques. Gradient elasticity was also used by Gutkin and Aifantis $/ 13 \mathrm{a}, \mathrm{b} /$ to eliminate the strain singularity and obtain the structure of the core of dislocation lines. A critical review of the special theory of gradient elasticity was provided by Altan and Aifantis /14/. Remarks concerning its relation to nonlocal theories and to the mixture theory of elastic phases are given. A uniqueness theorem is established, the nature of boundary conditions is discussed, Mode I, II, III crack problems are considered and wave propagation results are obtained. A nonlinear gradient elasticity theory was proposed by Triantafyllidis and Aifantis /15/ to discuss the localization of deformation in hyperelastic materials. Other types of gradient elasticity theory for small and large deformations are also available in unpublished form as a result of collaborative work between the last author and his co-workers $/ 10 \%$. This latter form has been shown to eliminate both stress and strain singularities at dislocation lines. In this connection, it is worth noting that the gradient elasticity theory was extended by Vardoulakis et al. /16/ to consider a surface energy term, which may not have an effect on the field equations, although it influences the boundary conditions. This enhanced theory was also used to solve the Mode III crack problem and reproduce a cusp-like profile for a clamped crack tip.

\subsection{Multifractal approach}

\subsubsection{Basic concepts}

Natural fractal sets show random self-similar morphologies, i.e. they look statistically the same by changing the scale of observation. In all natural fractal structures there is an upper and a lower bound limiting the scaling range; thus, a transition from the fractal (disordered) regime at the microscopic scale towards the Euclidean (homogeneous) regime at the largest scales takes place. The upper bound is represented by the macroscopic size of the set, while the lower bound is related to the size of the smallest measurable irregularities, e.g. aggregates in the case of concrete. The aforementioned transition was first pointed out by Mandelbrot $/ 17 /$, and later experimentally demonstrated, among others, by Carpinteri $/ 5 /$ and Carpinteri and Chiaia /18a,b/ in the case of concrete.

It is well-known that the nominal tensile strength of several materials undergoes very clear size effects. The usual trend is that of a strength decrease with the size, and this is more evident for disordered (i.e. macroscopically heterogeneous and/or damaged) materials. Griffith /19/ explained the strength size effect in the case of glass filaments, assuming the existence of inherent microcracks of a size proportional to the filament cross-section diameter. Weibull $/ 20 /$ gave a purely statistical explanation to the same phenomenon 
according to the weakest-link-in-a-chain concept. Only recently the two views have been harmonized, enriching the empirical approach of Weibull with the phenomenological assumption of Griffith. A statistical size distribution of self-similarity may be defined for which the most dangerous defect proves to be of a size proportional to the structural size. This corresponds to materials presenting a considerable dispersion in the statistical microcrack size distribution (disordered materials). In this case, the power of the linear elastic fracture mechanics (LEFM) stress singularity, $1 / 2$, turns out to be the slope of the strength versus size decrease in a bilogarithmic diagram. When the statistical dispersion is relatively small (ordered materials), the slope is less than $1 / 2$ and tends to zero for regular distributions (perfectly ordered materials) $/ 21 \%$

It is assumed that the reacting section or ligament of a disordered material at peak stress can be represented as a lacunar fractal space of dimension $2-d_{\sigma}$, with $0<d_{\sigma}<1$. This dimensional decrement may be attributed to the presence of voids and cracks and, in general, to any material inhomogeneity that leads to a cross-sectional weakening. On the other hand, the fracture surfaces of metals and concrete present an invasive fractal nature and have a dimension of $2+d_{G}$, where $0 \leq d_{G}<1$ is a dimensional increment representing the attenuation of fracture localization due to material heterogeneity and multiple cracking. These two assumptions lead to a renormalized tensile strength $\sigma_{L}^{*}$ and a renormalized fracture energy $G_{F}^{*}$ which are considered to be material constants $/ 21,22 \%$. Moreover, it was shown that the highest possible disorder in the microstructure is represented by a Brownian disorder, i.e. fractal dimensions equal to 1.5 and 2.5 seem to be respectively the lowest and the highest, for material ligaments and fracture surfaces, in the limit of microscopic scales of observation. The experimental determination of the size-effect exponents, either in the case of tensile strength (Carpinteri and Chiaia /18a, b/) or in the case of fracture energy (Carpinteri and Chiaia, /23/), whose absolute values have never been measured larger than 0.5 , is an indirect validation of the above assumption.

\subsubsection{Multifractal scaling laws for tensile strength and fracture energy}

Since the microstructure of a disordered material is the same independently of the macroscopic specimen size, the influence of disorder on the mechanical properties essentially depends on the ratio between a characteristic length $\ell_{c h}$ and the external size $d$ of the specimen. Thus, the effect of microstructural disorder becomes progressively less important at the largest scales, whereas it represents a fundamental feature at the smallest ones.

Based on the above assumption, two multifractal scaling laws (MFSL) have been proposed for tensile strength and fracture energy, respectively with the following analytical forms $/ 1,21,22 /$ (Fig. 1):

$$
\begin{aligned}
& \sigma_{u}(d)=f_{t}\left(1+\frac{\ell_{c h}}{d}\right)^{1 / 2}, \\
& G_{F}(d)=G_{F}^{\infty}\left(1+\frac{\ell_{c h}}{d}\right)^{-1 / 2} .
\end{aligned}
$$

In (1) and (2), $f_{t}, G_{F}^{\infty}, \ell_{c h}$ denote the lowest nominal tensile strength, the highest nominal fracture energy and the characteristic length, respectively. 


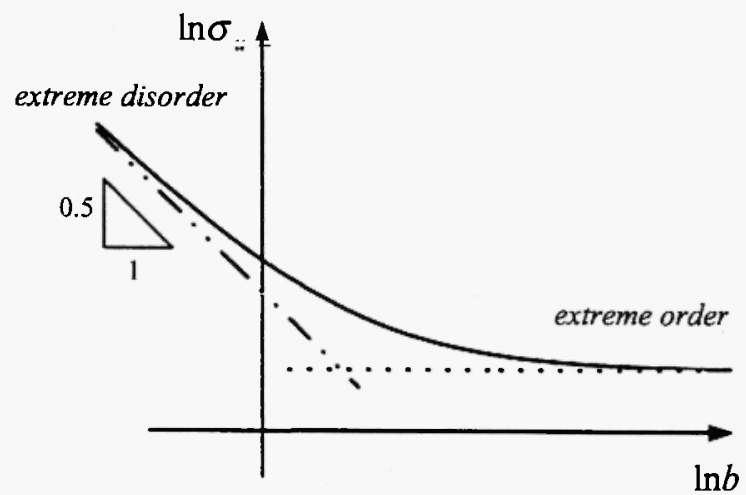

(a)

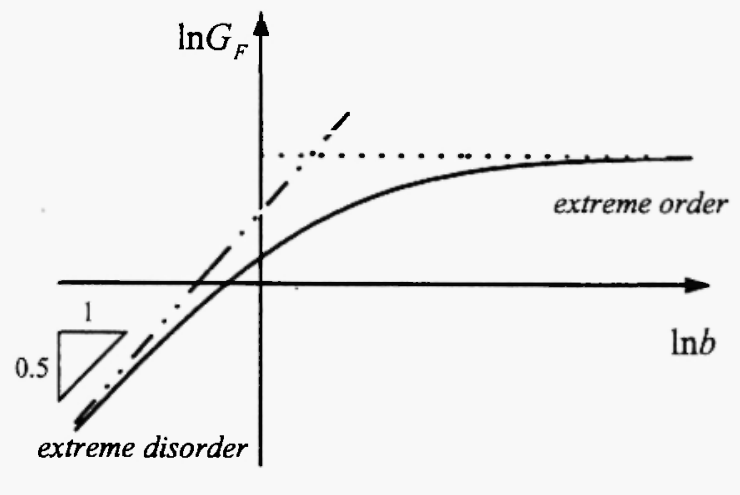

(b)

Fig. 1: Multifractal scaling laws (MFSL) for tensile strength (a) and fracture energy (b).

The asymptotic values of the two laws $\left(f_{t}, G_{F}^{\circ}\right)$ are reached only in the limit case of a specimen with infinite size, while the bracketed terms represent the variable influence of disorder on the mechanical behavior. It should be noted here that the bilogarithmic plots of these multifractal scaling laws show a slope of -0.5 and 0.5 in the limit of very small specimens, for tensile strength and fracture energy, respectively.

\section{SIZE EFFECT IN ELASTIC BOREHOLES}

The first attempt to interpret size effects in mechanical behavior of materials by employing gradient elasticity has been made by Aifantis $/ 24$. Size effects is a subject of increasing interest due to the fact that current applications in modern technology involve a variety of length scales ranging from centimeters (sheet metal forming) down to nanometers (thin film technology). This range of scales and the corresponding necessity for modeling and experiment have revealed that there is a strong connection between the various length scales involved and that the response may depend on size for otherwise geometrically similar specimens. In the simplest case of size-dependent response, one may consider the interaction between the length of the specimen and an internal length associated with the material microstructure. The interaction between macroscopic and microscopic length scales in the constitutive response and the corresponding interpretation of the associated size effects may be modeled through the introduction of higher order strain gradients in the respective constitutive equations $/ 24,29 /$.

It is recalled here that a well-known trend of brittle materials such as glass, ceramics, rocks, concrete and others is their tendency to become stronger as the cross-sectional area is decreased. One of the problems of great practical interest in the mining and petroleum industry, namely the size effect exhibited by borehole breakouts, was considered by using gradient elasticity /24/. Laboratory experiments conducted by various investigators in the past show that smaller holes are stiffer and fail at higher stresses than larger holes. One of 
the commonly reported outcomes of such tests is that the circumferential stress at the boundary of the borehole required to cause breakout is at least twice the unconfined compressive strength of the material.

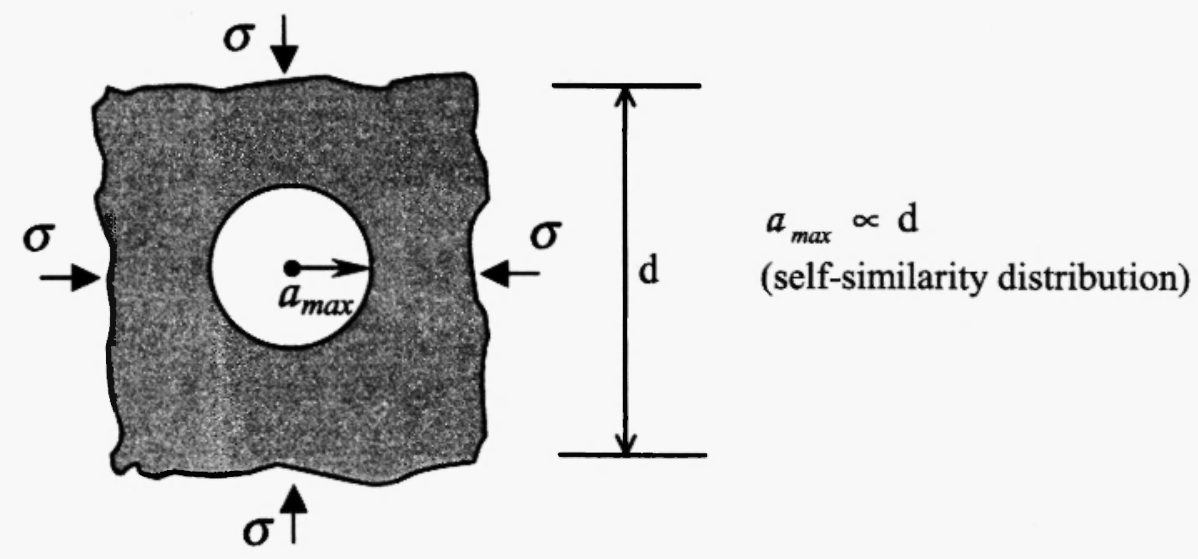

Fig. 2: Most dangerous hole of radius $a_{\max }$ in a disordered material subjected to uniform compressive load.

Structures with a multitude of cracks, voids or inclusions of a given size-distribution will be considered. The two assumptions made are: (a) the structure is macroscopically homogeneous and isotropic, and (b) the interaction between the imperfections is negligible. A set of geometrically similar structures containing many imperfections is considered, where $a_{\max }$ is the size of the most dangerous imperfection. If the maximum size $a_{\max }$ is proportional to the structural size $d$ (Fig. 2), then the strength size effect can be represented by the linear diagram In $\sigma_{j}$ versus Ind of LEFM /25/.

Next we consider a hollow elastic cylinder extending to infinity with a hole of radius, subjected to a uniform compressive stress $\sigma$ (Fig. 3). This is an idealization of the so-called borehole problem in geomechanics, where it has been observed that smaller holes are stiffer and fail at higher stresses. The problem was examined in the framework of a linear elastic gradient theory $/ 24,26 /$ and the experimental trends were compared with the obtained analytical solution. The failure stress decreases, and the stress concentration factor increases, as the radius of the borehole increases.

By using the following constitutive relationship of the gradient theory

$$
\sigma_{i j}=\lambda \varepsilon_{k k} \hat{o}_{i j}+2 \mu \varepsilon_{i j}-c \lambda \nabla^{2}\left(\varepsilon_{k k} \delta_{i j}\right)
$$

where $\left(\sigma_{i j}, \varepsilon_{i j}\right)$ are the stress and strain tensors, $(\lambda, \mu)$ the Lame's elastic constants, $\delta_{i j}$ denotes the unit tensor, $\nabla^{2}$ is the Laplacian operator and $c>0$ is the gradient coefficient, the corresponding expressions for stresses, strains and displacements have been found as follows $/ 26 /$ : 


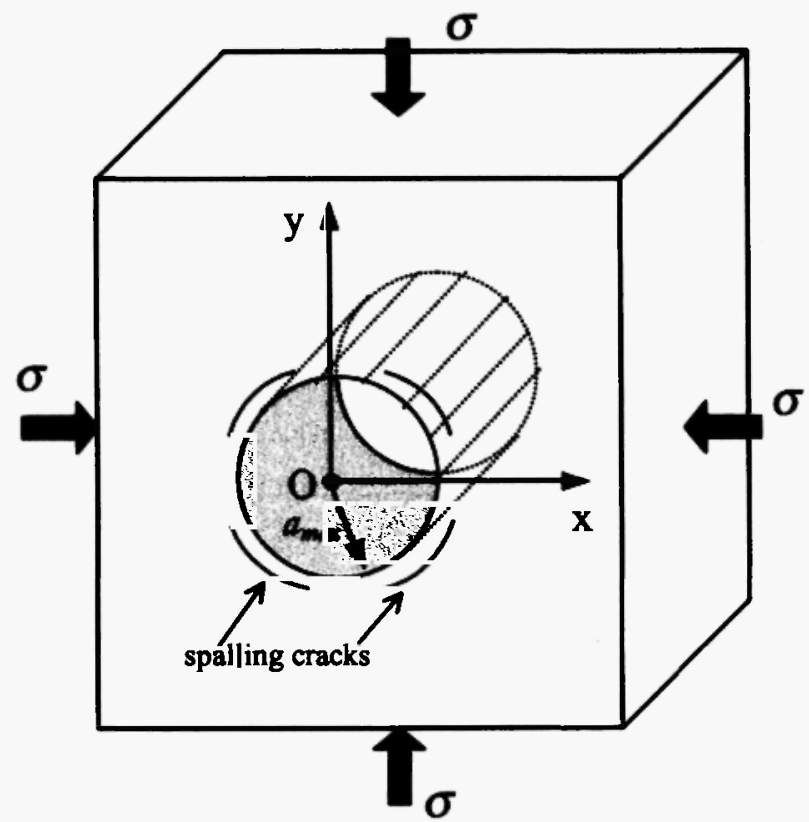

(a)

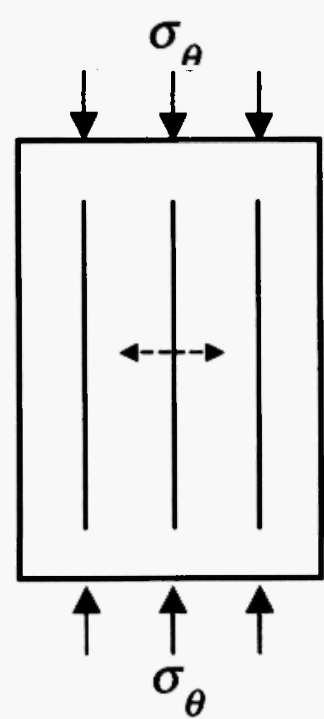

(b)

Fig. 3: (a) Circular borehole subjected to all-around uniform compression $\sigma$ and system of coordinates. Types of failure (splitting cracks causing spalling of hole edges) observed around circular openings in rocks; (b) Axial splitting crack pattern in uniaxial compression.

$$
\begin{aligned}
& \sigma_{r}=\sigma\left(1-\frac{a^{2}}{r^{2}}\right)+\frac{2 \sigma \sqrt{c^{\prime}}}{r T_{h}} F(r), \\
& \sigma_{\theta}=\sigma\left(1+\frac{a^{2}}{r^{2}}\right)-\frac{2 \sigma}{T_{h}}\left\{\frac{\sqrt{c^{\prime}}}{r} F(r)+K_{0}\left(\frac{r}{\sqrt{c^{\prime}}}\right)\right\}, \\
& \varepsilon_{r}=\frac{\sigma}{2 \mu}\left\{(1-2 v)-\frac{a^{2}}{r^{2}}+\frac{2 \sqrt{c^{\prime}}}{T_{h}}\left[\frac{1}{r} F(r)+\frac{1}{\sqrt{c^{\prime}}} K_{0}\left(\frac{r}{\sqrt{c^{\prime}}}\right)\right]\right\}, \\
& \varepsilon_{\theta}=\frac{\sigma}{2 \mu}\left\{(1-2 v)+\frac{a^{2}}{r^{2}}-\frac{2 \sqrt{c^{\prime}}}{r T_{h}} F(r)\right\}, \\
& u=\frac{\sigma}{2 \mu}\left\{(1-2 v) r+\frac{a^{2}}{r}-\frac{2 \sqrt{c^{\prime}}}{T_{h}} F(r)\right\},
\end{aligned}
$$

where $r \geq a, h=a / \sqrt{c^{\prime}}, T_{h}=h K_{1}(h)+K_{0}(h)$, and $F(r)=K_{1}\left(r / \sqrt{c^{\prime}}\right)-(a / r) K_{1}(h)$, while $K_{0}\left(r / \sqrt{c^{\prime}}\right)$, $K_{1}\left(r / \sqrt{c^{\prime}}\right)$ are second type modified Bessel functions of zero and first order, respectively. The expression for the circumferential stress $\sigma_{0}$ at the hole boundary is given by 


$$
\left.\sigma_{\theta}\right|_{r=a}=2 \sigma\left(1-\frac{K_{0}(h)}{T_{h}}\right), \quad c^{\prime}=\frac{v c}{1-v} .
$$

The stress concentration factor $K$ and the normalized failure stress $\sigma_{f}$ may be written as follows

$$
\left.K=2 \mid 1-\frac{K_{0}(h)}{h K_{1}(h)+K_{0}(h)}\right), \quad \sigma_{f}=\frac{1}{2}\left(1+\frac{K_{0}(h)}{h K_{1}(h)}\right) .
$$

\subsection{Stress concentration factor}

The logarithmic form of the first equation in (10) is

$$
\ln K=\ln 2+\ln h+\ln \left(K_{1}(h)\right)-\ln \left[h K_{1}(h)+K_{0}(h)\right]
$$

(a) Large holes: This means that $\ln h \rightarrow \infty$, so that $h=a / \sqrt{c^{\prime}}$ tends to $\infty$, where $a \gg \sqrt{c^{\prime}}$. It follows that $\ln K=\ln 2=0.693$ or $K=2$. For large values of dimensionless hole radius $h$, the gradient solution coincides with the classical solution. The behavior of the hole is not sensitive to the material microstructure and the mechanical response is as the classical one.

(b) Holes with radius comparable with the gradient coefficient: In this case we have that $\operatorname{In} h=0$, so that $h=a / \sqrt{c^{\prime}}$ is equal to one, where $a=\sqrt{c^{\prime}}$. Consequently, $\ln K=0.163$ or $K=1.177$. When the dimensionless hole radius $h$ approaches the square root of the gradient coefficient, $\sqrt{c^{i}}$ (material constant), the maximum stress at the edge of the hole is 1.177 times larger than that applied externally. The strength of the plate of dimensions much larger than the hole is reduced to $\sim 85 \%$ of that of the intact plate. Classical elasticity $/ 22$ predicts that this reduction is equal to $50 \%$ regardless of the hole radius $h$.

(c) Small holes: In this case we have that $\ln h \rightarrow-\infty$, so that $h=a / \sqrt{c^{\prime}}$ tends to 0 , where $a \ll \sqrt{c^{\prime}}$ : $\lim _{h \rightarrow 0} K=0$. Without the hole, the applied stress distributes in the whole plate with the value of $\sigma$. From the physical point of view this means that $K$ must not be less than one and that the minimum stress concentration factor is equal to one.

(d) We will now determine the lowest value of the dimensionless hole radius for which the stress concentration factor assumes the minimum value. For $K-1$ or $\ln K=0$ we have $h=a / \sqrt{c^{\top}} \cong 0.6$ or $\ln h \approx-0.511$. For $a \approx 0.6 \sqrt{c^{\prime}}$ the stress concentration factor $K$ takes its lowest value i.e. one. It can be asserted that for $h \leq 0.6$ the stress concentration factor remains constant and equal to one. For dimensionless hole radii less than approximately 0.6 there is no (size) effect on the material behavior. The previous results are shown in Table 1 and illustrated in a bilogarithmic diagram in Fig. 4. It is observed that, for a hole radius $a \geq 10 \sqrt{c^{\prime}}$, gradient elasticity tends to coincide with classical elasticity. 
Vol. 12, No. 2, 2001

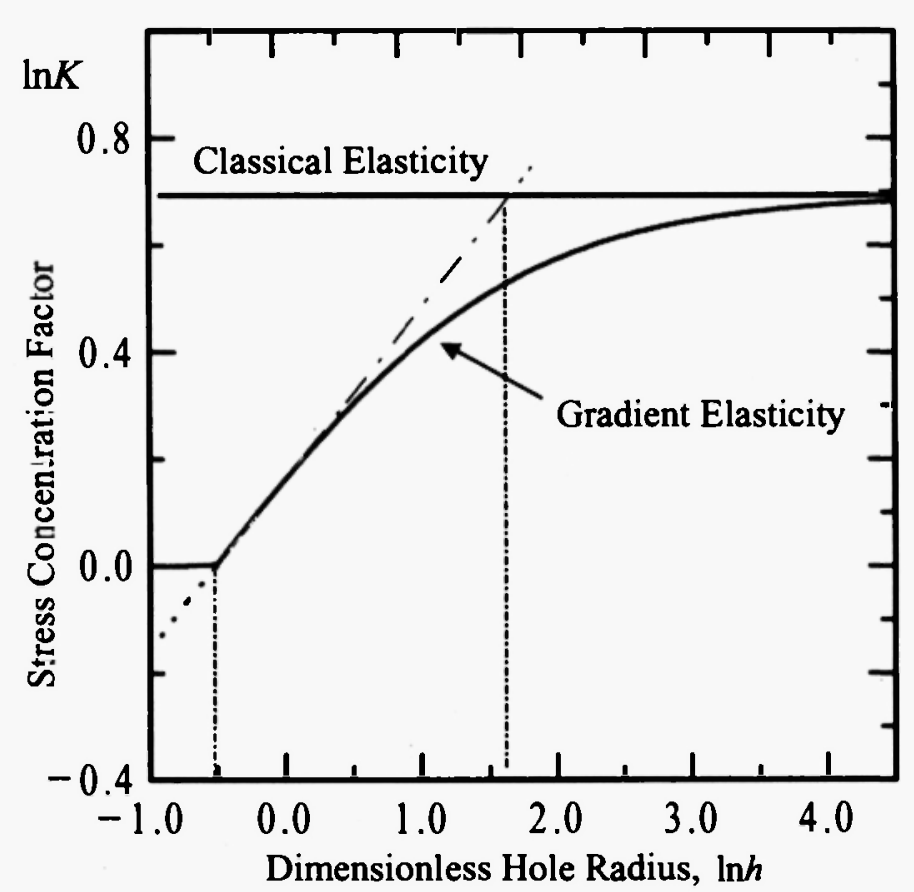

Fig. 4: Bilogarithmic plot of stress concentration factor versus dimensionless hole radius.

\section{Table I}

\begin{tabular}{l|ccccc}
\hline$a$ & $\ll \sqrt{c^{\prime}}$ & $\cong 0.6 \sqrt{c^{\prime}}$ & $\sqrt{c^{\prime}}$ & $\cong 5.27 \sqrt{c^{\prime}}$ & $\gg \sqrt{c^{\prime}}$ \\
\hline$H$ & 0 & $\cong 0.6$ & 1 & $\cong 5.27$ & $\infty$ \\
$K$ & $\ldots 1 .$. & 1 & 1.177 & $\cong 1.704$ & 2 \\
\hline
\end{tabular}

\subsection{Normalized failure stress}

Similarly, the logarithmic form of the second equation in (10) is

$$
\ln \sigma_{f}=-\ln 2-\ln h-\ln \left(K_{1}(h)\right)+\ln \left[h K_{1}(h)+K_{0}(h)\right]
$$

For large holes $\ln h \rightarrow \infty$, so that $h=a j \sqrt{c^{j}}$ tends to $\infty$, where $a \gg \sqrt{c^{\prime}}$. Then, $\ln \sigma_{f}=-\ln 2=-0.693$ and $\sigma_{f}=0.5$. For large values of the dimensionless hole radius $h$, we obtain the same result as in the previous case, i.e. the gradient solution coincides with the classical solution.

If the hole radius is comparable with the gradient coefficient $c^{\prime}$, we have $\ln h=0$ or $h=a / \sqrt{c^{\prime}}=1$ or $a=\sqrt{c^{\prime}}$. Then $\ln \sigma_{f}=-0.163$ or $\sigma_{f}=0.849$. For small holes, we have $\ln h \rightarrow-\infty$ or $h=a i \sqrt{c^{\prime}} \rightarrow 0$, so that $a \ll \sqrt{c^{\prime}}$. Then. $\lim _{h \rightarrow 0} \sigma_{f}=\infty$. For hole radii tending to zero, $\sigma_{f}$ takes unrealistic values tending to infinity. Physically, this cannot be true and it is concluded that the maximum value of the normalized failure 
stress is one. The smallest hole radius for which the normalized failure stress takes its maximum value, is found to be $a \simeq 0.6 \sqrt{c^{\prime}}$. For $h \leq 0.6$, the normalized failure stress remains constant and equal to one. The previous results are shown in Table 2 and illustrated in a bilogarithmic diagram in Fig. 5.

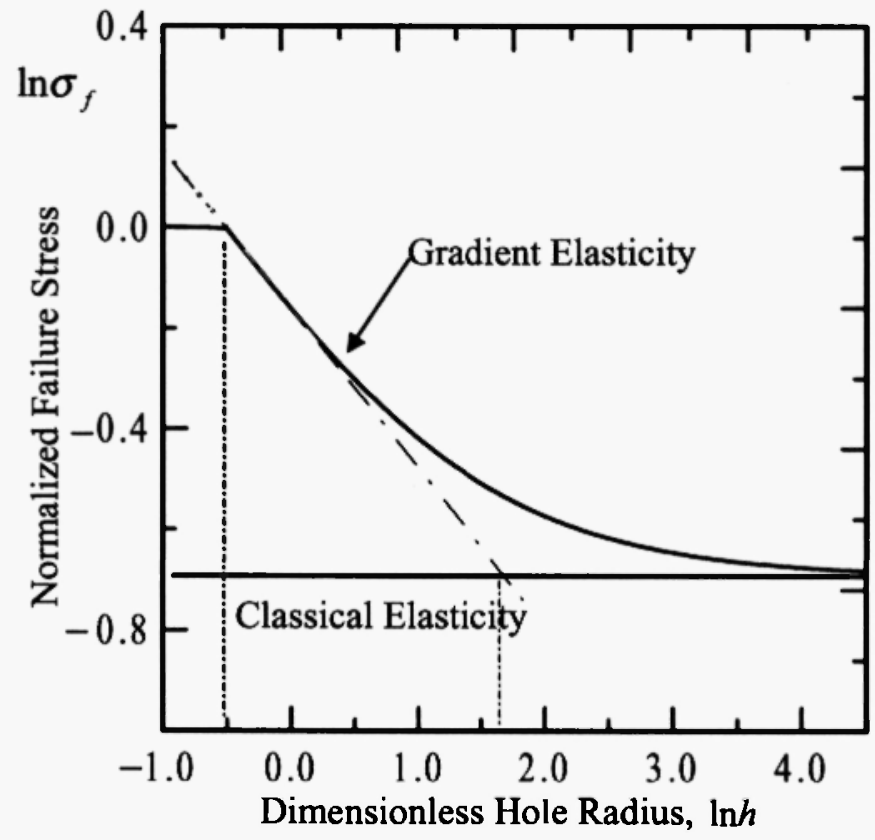

Fig. 5: Bilogarithmic plot of normalized failure stress versus dimensionless hole radius.

Table 2

\begin{tabular}{l|ccccc}
\hline$a$ & $\ll \sqrt{c^{\prime}}$ & $\cong 0.6 \sqrt{c^{\prime}}$ & $\sqrt{c^{\prime}}$ & $\cong 5.27 \sqrt{c^{\prime}}$ & $\gg \sqrt{c^{\prime}}$ \\
\hline$h$ & 0 & $\simeq 0.6$ & 1 & 5.27 & $\infty$ \\
$\sigma_{f}$ & $.1 .$. & 1 & 0.849 & 0.587 & 0.5 \\
\hline
\end{tabular}

The slope of the curve which represents the variation of the normalized failure stress versus the dimensionless hole radius is given by the following expression,

$$
\frac{\partial\left[\ln \sigma_{f}\right]}{\partial[\ln h]}=-1+\frac{K_{0}(h)\left[h K_{0}(h)+K_{1}(h)\right]}{K_{1}(h)\left[h K_{1}(h)+K_{0}(h)\right]} .
$$

Fig. 5 depicts that, for large values of $h$, the present gradient model provides similar results as the multifractal scaling law proposed by Carpinteri $/ 1,21 /$ and that the slope of the diagram tends to zero. Large holes show a residual normalized failure stress, which is the same as that predicted by classical elasticity. For 
$h \geq 10$ or $a \geq 10 \sqrt{c^{\prime}}$, the gradient approach does not have any influence in the borehole problem. On the other hand, when $h$ approaches small values, it is remarkable to notice the significant increase in the apparent strength. The slope in this case is approximately equal to -0.325 . The corresponding size effect observed deals with the microstructure of the material. When the hole radius is comparable with the square root of the gradient coefficient, $\sqrt{c^{\prime}}$ (material parameter), the stress concentration (and size) effect vanishes. On the other hand, in the case that the hole radius is very large as compared with $\sqrt{c^{\prime}}$, gradient elasticity provides the classical solution.

\section{COMPARISON BETWEEN MULTIFRACTAL SCALING LAW (MFSL) AND GRADIENT ELASTICITY}

Both theories consider the occurrence of the microstructure in the material failure behavior and the size dependence of the mechanical behavior. They lead to similar size effects, i.e. the larger the structural size, the lower the strength becomes. From the solution of the borehole problem it has been observed that the maximum stress at the edge of the hole is lower when the hole radius decreases.

Gradient theory accounts for the effect of size in the expression for the stress concentration factor and the normalized failure stress through the introduction of the Laplacian of volumetric strain in Hooke's law. This was motivated by the occurrence of the material microstructure (imperfections or irregularities), as in the multifractal approach. The size effect in the bilogarithmic plane $\ln \sigma_{f}$ vs $\ln h$ is nonlinear and shows an upward concavity according to the multifractal scaling law, as well as to the gradient approach. The two approaches predict also an asymptotic value of the strength (classical elasticity) for large structural sizes. For small structural sizes both theories lead to similar results, i.e. increase of strength as the structural size becomes smaller up to the intrinsic strength of the material.

Acknowledgements: The financial support of the European Commission under the TMR Project Grant No. FMRXCT960062 and the LISSAC Project No. FIKS-CT1999-00012 is gratefully acknowledged.

\section{REFERENCES}

1. Carpinteri, A.: Strength and toughness in disordered materials: Complete and incomplete similarity, In: Size-Scale Effects in the Failure Mechanisms of Materials and Structures (Carpinteri, A., ed.), E \& FN Spon, 2-6 Boundary Row, London, pp. 3-26, 1996.

2. Nemat-Nasser, S. and Hori, M.: Micromechanics: Overall properties of heterogeneous materials, North-Holland, Amsterdam, 1993. 
3. Carpinteri, A., Chiaia, B. and Nemati M. N.: Complex fracture energy dissipation in concrete under different loading conditions, Mechanics of Materials, vol. 26, pp. 93-108, 1997.

4. Mandelbrot, B. B.: The fractal geometry of nature, W. H. Freeman and Company, New York, 1982.

5. Carpinteri, A.: Fractal nature of material microstructure and size effects on apparent mechanical properties, Mech. of Materials, vol. 18, pp. 89-101, 1994.

6. Aifantis, E.C.: On the microstructural origin of certain inelastic models, J. Mat. Engng. Tech., vol.106, pp. 326-330, 1984.

7. Aifantis, E.C.: The physics of plastic deformation, Int. J. Plasticity, vol. 3, pp. 21 1-247, 1987.

8. Aifantis, E.C.: On the role of gradients in the localization of deformation and fracture, Int. J. Engng. Sci., vol. 30, pp. 1279-1299, 1992.

9. Altan, B. S. and Aifantis, E.C.: On the structure of mode III crack tip in gradient elasticity, Scripta Met., vol.26, pp. 319-324, 1992.

10. Ru, C.Q. and Aifantis, E.C.: A simple approach to solve boundary-value problems in gradient elasticity, Acta Mechanica, vol.101, pp. 59-68, 1993.

11. Unger, J. and Aifantis, E.C.: The asymptotic solution of gradient elasticity for Mode III, Int. J. Fract. 71, R27-R32, 1995.

12. Exadaktylos, G. E. and Aifantis, E.C.: Two and three dimensional crack problems in gradient elasticity, J. Mechanical Behavior 7, 93-117, 1996.

13. a) Gutkin, M. Y. and Aifantis, E.C.: Screw dislocation in gradient elasticity, Scripta Mater., vol.35, pp. 1353-1358, 1996; b) Gutkin, M. Y. and Aifantis, E.C.: Edge dislocation in gradient elasticity, Scripta Mater., vol.36, pp. 129-135, 1997.

14. Altan, B. S. and Aifantis, E.C.: On some aspects in the special theory of gradient elasticity, $J$. Mechanical Behavior., vol.8, pp. 231-282, 1997.

15. Triantafyllidis, N. and Aifantis, E.C.: A gradient approach to localization of deformation - I. Hyperelastic materials, J. of Elasticity, vol. 16, pp. 225-237, 1986.

16. Vardoulakis, I., Exadaktylos, G. E. and Aifantis, E. C.: Gradient elasticity with surface energy: ModeIII crack problem, Int. J. Solids Structures, vol. 33, pp. 4531-4559, 1996.

17. Mandelbrot, B. B.: Self-affine fractals and fractal dimension, Phys. Scr., vol. 32, pp. 257-260, 1985.

18. a) Carpinteri, A., and Chiaia, B.: Multifractal nature of concrete fracture surfaces and size effects on nominal fracture energy, Mater. Struct., vol. 28, pp. 435-443, 1995; b) Carpinteri, A., and Chiaia, B.: Crack-resistance behavior as a consequense of self-similar fracture topologies, Int. J. Fract., vol. 76, pp. 327-340, 1995.

19. Griffith, A. A.: The phenomena of rupture and flow in solids, Phil. Trans. Roy. Soc. (London) Ser. A 221, pp. 163-198, 1920.

20. Weibull, W.: A statistical theory of the strength of materials, Swedish Royal Institute for Engineering Research, Stockholm, 1939. 
21. Carpinteri, A.: Scaling laws and renormalization groups for strength and toughness of disordered materials, Int. J. Solids Structures, vol. 31, pp. 291-302, 1994.

22. Carpinteri, A., and Chiaia, B.: Multifractal scaling laws in the breaking behavior of disordered materials, Chaos, Solitons \& Fractals, vol. 8, pp. 135-150, 1997.

23. Carpinteri, A., and Chiaia, B.: Size effects on concrete fracture energy: Dimensional transition from order to disorder, Mater. Struct, vol. 29, pp. 259-266, 1996.

24. Aifantis, E.C.: Higher order gradients and size effects, In: Size-Scale Effects in the Failure Mechanisms of Materials and Structures (Carpinteri, A., ed.), E \& FN Spon, 2-6 Boundary Row, London, pp. 231242, 1996.

25. Carpinteri, A.: Mechanical Damage and Crack Growth in Concrete - Plastic Collapse to Brittle Fracture, Martinus Nijhoff Publishers, Dordrecht, 1986.

26. Efremidis, G. T., Ning, J., Exadaktylos, G. E. and Aifantis, E. C.: Gradient elasticity and size effects, $5^{\text {th }}$ National Greek Congress on Mechanics, vol. 2, Ioannina, Greece, pp. 915-920, 1998.

27. Carpinteri, A.: Structural Mechanics - A Unified Approach, E \& FN Spon, London, 1997.

28. Timoshenko, S. P. and Goodier, J. N.: Theory of Elasticity, McGraw-Hill, New York, 1970.

29. Bazant, Z. P., Kim, J.-J. H., Daniel, I. M., Becq-Giraudon, E. and Zi, G.: Size effect on compression strength of fiber composites failing by kink band propagation, Int. J. of Fracture, vol.95, pp. 103-141, 1999. 